\title{
Risk of incidental genital tract malignancies at the time of myomectomy and hysterectomy for benign conditions
}

\author{
Garima Yadav, MS ${ }^{1}$, Meenakshi Rao, MD², Shuchita Batra Goyal, MD', Pratibha Singh, MD1, \\ Priyanka Kathuria, $\mathrm{MS}^{1}$, Meenakshi Gothwal, MD' \\ Departments of ${ }^{1}$ Obstetrics and Gynecology, ${ }^{2}$ Pathology, All India Institute of Medical Sciences, Jodhpur, India
}

\section{Objective}

To determine the incidence of accidentally diagnosed genital malignancies in women undergoing gynecological surgeries for pathologies presumed to be benign.

\section{Methods}

Data of all women who underwent hysterectomy for benign indications were retrieved from hospital records. A total of 628 cases of hysterectomy and 35 cases of myomectomy were included. The final histopathology was accessed through the computer records, and the total number of genital tract malignancies was included.

\section{Results}

The incidence of occult uterine, ovarian, and cervical malignancies in women undergoing hysterectomy was $0.47 \%$, $0.31 \%$, and $0.15 \%$, respectively. The incidence of uterine corpus malignancies in women undergoing myomectomy was $2.8 \%$, and 3 cases of smooth muscle tumor of uncertain malignant potential (STUMP) were also identified. Endometrial stromal sarcomas were the most common pathology among uterine malignancies, followed by leiomyosarcoma and endometrial adenocarcinoma.

\section{Conclusion}

Postoperative diagnosis of malignancies in women undergoing surgery for benign indications poses great challenges to the treating surgeon. Occult malignancies must be ruled out by thorough preoperative assessment, especially in some subsets of women. Morcellation is an inevitable technique and complement to laparoscopic surgeries, although little chances of malignancy spread is related to its use. Nevertheless, thorough preoperative investigations and always ensuring contained morcellation can minimize these chances.

Keywords: Genital neoplasms; Hysterectomy; Myomectomy; Leiomyosarcoma

\section{Introduction}

Hysterectomy is one of the most common surgical procedures performed in gynecology, and often, it is performed as a benign indication. Common indications are abnormal uterine bleeding (AUB), uterine fibroids, adenomyosis, endometriosis, pelvic organ prolapse, postmenopausal bleeding (PMB), and benign adnexal masses $[1,2]$. These benign pathologies can be reliably picked up by ultrasound, preferably done through both transvaginal and transabdominal routes. Most gynecologists do not resort to advanced imaging modalities as a routine during preoperative workups. However, postoperative diagnosis of genital malignancies in women operated for benign-like pathologies may create clinical di- lemmas in the mind of treating physicians and worsens the oncological outcomes of these patients. When malignancy

Received: 2020.07.13. Revised: 2020.10.06. Accepted: 2020.10.25. Corresponding author: Garima Yadav, MS

Department of Obstetrics and Gynecology, All India Institute of Medical Sciences, Basni, Phase 2, Jodhpur, Rajasthan 342001, India E-mail: garimapunein@gmail.com

https://orcid.org/0000-0002-1268-6973

Articles published in Obstet Gynecol Sci are open-access, distributed under the terms of the Creative Commons Attribution Non-Commercial License (http://creativecommons. org/licenses/by-nc/3.0/) which permits unrestricted non-commercial use, distribution, and reproduction in any medium, provided the original work is properly cited.

Copyright (c) 2020 Korean Society of Obstetrics and Gynecology 


\section{Obstetrics \& Gynecology Science}

Vol. 64, No. 2, 2021

is suspected in the preoperative workup, more extensive staging and cytoreductive procedures should be performed in addition to uterus removal. Three methods of performing hysterectomy are abdominal, laparoscopic, and vaginal. The opted route of surgery mainly depends on the size of the uterus or specimen, the nature of pathology, whether benign or malignant, and the operating surgeon's experience.

In the past two decades, laparoscopy has become the preferred route of hysterectomy because of its apparent advantages, but it might require a procedure such as power morcellation to remove larger uteri. Laparoscopic power morcellator has been used for over 25 years to extract large specimens of fibroids or adnexal masses. However, there have been reports of intraperitoneal seeding of benign and malignant diseases, such as leiomyoma, endometriosis, and unsuspected ovarian or uterine malignancy [3]. This creates problems in the accurate grading and staging of sarcoma. Moreover, prognosis of such patients is poor after the malignant tissue has dissipated in the abdominal cavity. Due to these concerns, the use of laparoscopic power morcellator was banned by the U.S. Food and Drug Administration (USFDA) on November 24, 2014, in most patients who underwent myomectomy and hysterectomy for fear of dissemination of unrecognized malignancy [4].

Most gynecological cancers are picked up preoperatively owing to their complex symptoms and available investigations. However, there are no reliable diagnostic markers for leiomyosarcoma, asymptomatic endometrial cancer, and lowgrade or borderline ovarian malignancies [5]. The reported incidence of occult uterine malignancies ranges from $0 \%$ to $3.17 \%$, and limited data is available on the incidence of occult cervical and ovarian malignancies with reported incidences of $0.6 \%$ and $0.19 \%$, respectively [6-9]. In this study, we describe the incidence of postoperatively diagnosed genital tract malignancies involving the uterus, fallopian tube, ovary, and cervix, along with the management and follow-up of these accidentally diagnosed cancers. It is also imperative to determine the lacunas in the preoperative workups of a particular subset of women likely to be diagnosed with accidental malignancies and the safest route of surgery to prevent such unhappy encounters in these presumably high-risk women.

\section{Materials and methods}

This retrospective study was conducted in the Department of Obstetrics and Gynecology at All India Institute of Medical Sciences, Jodhpur, from 2017 to 2019. All women who underwent hysterectomies for non-malignant causes were included. A total of 628 cases of hysterectomy performed through laparoscopic, abdominal, and vaginal routes, along with 35 cases of laparoscopic and open myomectomies, were included in the study. The study was approved by the institutional ethical committee.

All women with heavy menstrual bleeding (HMB) owing to a non-malignant cause, prolapse uterus, unexplained PMB, fibroid uterus, and adenomyosis not responding to medical management were included in the study. All patients were evaluated preoperatively by the surgeon, and the surgical route was decided. All women underwent preoperative pap smears. Endometrial biopsy was available for all cases of $H M B$, where the causes of bleeding were not obvious. Endometrial biopsy was performed using endometrial biopsy curette (Novak's curette) in most cases, and in the remaining patients when the biopsy report was inconclusive or in women presenting with PMB, hysteroscopy was performed to take an endometrial biopsy. Pelvic ultrasound (USG) was available for all women included in the study, and no malignancy was suspected on USG in all included cases. All women undergoing laparoscopic myomectomy or conservative surgery were benign-like ovarian cysts $(>10 \mathrm{~cm})$, who underwent T2-weighted magnetic resonance imaging (MRI) to rule out any malignancy. This data was collected by the electronic patient management system of the hospital. Women with preoperative suspicion of malignancy, those wherein data collection was inadequate, or missing follow-up data were excluded from the analysis. The preoperative assessment, intraoperative findings, type of surgery, final histopathological reports, type of adjuvant therapy, and follow-up of all the diagnosed malignancies were assessed.

The staging was retrospectively revised based on surgical records and pathologic results. The primary outcome was to determine the incidence of occult genital malignancies diagnosed in women undergoing hysterectomy with or without salpingo-oophorectomy for benign indications and women undergoing myomectomy uterine or cervical fibroids. We compared the incidence of unexpected uterine malignancies and cervical and ovarian malignancies in the laparoscopic, 


\section{Obstetrics \& Gynecology Science}

Garima Yadav, et al. Incidental genital tract malignancies

abdominal, and vaginal routes.

\section{Results}

A total of 628 hysterectomies and 35 myomectomies were included in the study because their detailed preoperative workup, intraoperative findings, histopathology report, and follow-up was available. The median age for women undergoing hysterectomy was 46 years (range, 38-72 years), and those undergoing myomectomies was 34 years (range, 24-42 years). The most common indication for hysterectomy was AUB (45.38\%), while the most common cause was ovulatory dysfunction leading to simple endometrial hyperplasia and adenomyosis. Nearly $30 \%$ of women underwent uterovaginal prolapse, and most of these women were postmenopausal. Large and symptomatic fibroid uterus was the cause for surgery in $18.78 \%$ of women, and whenever fertility was not desired, a myomectomy was performed. Among the 2.8\% pan-hysterectomies performed for benign-like adnexal masses, endometriosis was the most common pathology, followed by large simple ovarian cysts in peri-menopausal females. Approximately $1 \%$ of women underwent surgery for persistent unexplained PMB after ruling out endometrial malignancy by diagnostic hysteroscopy and directed biopsies (Table 1). Among the 35 women who underwent myomec-

Table 1. Indications for hysterectomy with or without salpingooophorectomy

\begin{tabular}{lcc}
\hline Symptom No. & Indication & $\begin{array}{c}\text { No. of cases } \\
\text { (\% of total) }\end{array}$ \\
\hline 1 & $\begin{array}{c}\text { Abnormal uterine bleeding } \\
\text { (other than abnormal uterine } \\
\text { bleeding due to leiomyomatosis) }\end{array}$ & $285(45.38)$ \\
\hline 2 & Prolapse uterus & $192(30.57)$ \\
3 & Symptomatic fibroid uterus & $118(18.79)$ \\
4 & Benign adnexal masses & $18(2.87)$ \\
6 & Postmenopausal bleeding & $6(0.96)$ \\
7 & Cervical fibroid & $3(0.48)$ \\
8 & HSIL following LEEP & $1(0.16)$ \\
9 & Post- menopausal dermoid cyst & $1(0.16)$ \\
10 & Recurrent pyometra & $4(0.64)$ \\
& Total & $628(100.00)$ \\
\hline
\end{tabular}

HSIL, high-grade squamous intraepithelial lesion; LEEP, loop electrosurgical excision procedure. tomy, $80 \%$ had symptomatic intramural fibroids, $11.4 \%$ had cervical fibroids, and $8.6 \%$ had fibroids impinging on the endometrial cavity.

Vaginal hysterectomy $(\mathrm{VH})$ was the most common route of surgery $(33.7 \%)$, preferred to treat prolapse cases, and AUB cases with small uniform uteri with no associated adnexal pathology. Total laparoscopic hysterectomy (TLH) was performed in $31.21 \%$ of the cases, followed by abdominal hysterectomy (30.09\%). The abdominal route was opted in women with uterine size $>14$ weeks, co-existing ovarian cyst, cervical or broad ligament fibroids, and in case of a contraindication to general anesthesia or pneumoperitoneum, as in patients with compromised cardio-respiratory function. Robotic hysterectomy was one of the surgery routes, although only $4.9 \%$ and the most common pathology was AUB (Table 2). All specimens in laparoscopic and robotic cases were retrieved vaginally through the vault opening using techniques such as coring and bisection, and power morcellation was not performed in any case. In the case of myomectomies, $94.2 \%$ (33 out of 35 women) underwent myomectomy through the minimal access technique (laparoscopic or robotic). In all of these women, power morcellation in a contained bag was performed to retrieve the fibroids.

A total of 10 cases of occult genital malignancies were

Table 2. Different modes and types of surgery

\begin{tabular}{lrc}
\hline Symptom No. & Type of surgery & No. of cases \\
\hline 1 & Vaginal hysterectomy & 212 \\
2 & Abdominal hysterectomy & 189 \\
3 & Total laparoscopic hysterectomy & 196 \\
4 & Robot-assisted hysterectomy & 31 \\
5 & Laparoscopic myomectomy & 33 \\
6 & Open myomectomy & 2 \\
\hline
\end{tabular}

Table 3. Various occult genital malignancies diagnosed postoperatively following hysterectomy and myomectomy

\begin{tabular}{lcc}
\hline Symptom No. & Case & $\begin{array}{c}\text { Total No. of } \\
\text { cases }\end{array}$ \\
\hline 1 & Uterine corpus & 4 \\
2 & $\begin{array}{c}\text { Smooth muscle tumor of } \\
\text { unknown malignant potential }\end{array}$ & 3 \\
3 & Cervix (gastric type) & 1 \\
4 & Ovarian malignancy & 2 \\
\hline
\end{tabular}




\section{Obstetrics \& Gynecology Science}

Vol. 64, No. 2, 2021

detected in our study population undergoing hysterectomy or myomectomy (Table 3). Among these 10 cases, we found six cases of genital tract malignancy in women who underwent hysterectomy with or without salpingo-oophorectomy, three cases of smooth muscle tumor of uncertain malignant potential (STUMP), and one case of endometrial stromal sarcoma (ESS) in women who underwent myomectomy. Among women undergoing hysterectomy, the incidence of occult malignancy of the uterine corpus was $0.47 \%$, the incidence of ovarian malignancy was $0.31 \%$, and the incidence of cervical malignancy was lowest $(0.15 \%)$. Notably, occult malignancy incidence was $2.8 \%$ in women undergoing myomectomy, and the incidence increased to $11.4 \%$ if cases of STUMP were included.

We also observed that the incidence of uterine malignancy was higher with abdominal hysterectomy than with the laparoscopic route (1.05\% compared to $0 \%)$, and all cases with occult malignancy in myomectomies were operated laparoscopically. Approximately $87.5 \%$ of corpus uteri malignancy cases were stage I, and all the remaining cases of ovarian and cervical malignancies were also in the early stage (Stage la and Stage Ib, respectively). Only one case of leiomyosarcoma was upstaged to stage IIlb following re-surgery. Nine out of the 10 cases remained disease-free following postoperative management, and 1 patient expired at the ninth month of follow-up secondary to a cardio-vascular accident.

\section{Discussion}

The overall incidence of occult genital malignancies in women undergoing hysterectomy in our population was $0.95 \%$, and that in women undergoing myomectomy was $2.8 \%$. Interestingly, the incidence of occult malignancies diagnosed following hysterectomy for presumed benign pathologies was lower than that reported in the recent literature, but the incidence was higher in the myomectomy group [9]. Nearly $67 \%$ of the women with occult uterine, cervical, and ovarian malignancies diagnosed following hysterectomy were postmenopausal in the absence of any clinical suspicion of malignancy based on history, examination, or preoperative imaging. Before any surgery involving removal of the uterus with or without removal of the adnexa, the recommended preoperative workup included a cervical smear, an endometrial biopsy (in the case of AUB and PMB), and a detailed
USG. Advanced imaging techniques like contrast-enhanced computed tomography (CECT) and Diffusion-weighted/T2 weighted magnetic resonance imaging (MRI) were not routinely done for pelvic pathologies that appeared benign on USG. The restricted use of MRI/CECT can be explained by the fact that the diagnostic accuracy of transvaginal ultrasound (TVS) is higher for benign pelvic pathologies. It is a more accessible, affordable, and safe imaging tool than CECT and MRI.

Among the uterine corpus malignancies, three STUMP cases were diagnosed postoperatively in women who underwent laparoscopic myomectomy for symptomatic intramural fibroids in the reproductive age group. Preoperative USG and MRI did not report any suspicion of malignancy. The mitotic count ranged from 10-20/hpf and tumor cells were positive for vimentin, along with estrogen and progesterone receptors. One patient with a mitotic count $>20 / \mathrm{hpf}$ underwent a second surgery in the form of total abdominal hysterectomy and bilateral salpingo-oophorectomy. The final histopathology also confirmed the diagnosis of STUMP. The patients underwent baseline chest, abdomen, and pelvic CT scans postoperatively, which showed no metastatic lesion. All patients were disease-free at the end of 4-24 months of follow-up. Adjuvant chemotherapy or hormonal therapy had no role in women with STUMP, but the recurrent disease required surgical excision followed by hormonal therapy with medroxyprogesterone acetate (MPA) gonadotropin releasing hormone agonists and/or chemotherapy [10-12]. Morcellation of such tumors should be ideally avoided if there is a preoperative suspicion; otherwise, it can lead to diffuse peritoneal implants that may be benign or malignant [13] In our population, the incidence of STUMP was $8.5 \%$, and all the fibroids were large $(>7 \mathrm{~cm})$. Hence, it is important to conclude that in larger fibroids, the operating team must anticipate such pathologies and take all measures to avoid spillage of tumor bits in the peritoneal cavity. These women must be strictly followed up as recurrence occurs in $11 \%$ of these women [14].

The incidence of malignancy of the uterine corpus in women undergoing hysterectomy was $0.47 \%$ and that during myomectomy, it was $2.8 \%$. These numbers contrast to those reported by Desai et al. [9] that occult uterine corpus malignancies were seen in $1.44 \%$ of women undergoing hysterectomy and $0.21 \%$ in myomectomy specimens, although the above study did not mention the histopathological types 


\section{Obstetrics \& Gynecology Science}

of malignancies identified. In our study, three women were identified with malignancy of the uterine corpus and the pathologies were uterine leiomyosarcoma (LMS), ESS, and endometrial adenocarcinoma. All the malignancies were stage IB, as diagnosed following histopathological report. The endometrial adenocarcinoma patient had undergone total abdominal hysterectomy (TAH) with bilateral salpingooophorectomy (BSO) in view of PMB and a fibroid polyp in the uterus, which did not reveal malignancy on preoperative biopsy or USG. The patient with LMS was peri-menopausal and was planned for TAH in view of a large fibroid $(10 \mathrm{~cm})$; however, intraoperatively, the fibroid's gross appearance was suspicious; hence, BSO was also performed. This patient had a preoperative CECT of the pelvis, which raised suspicion of a complex ovarian mass, but the preoperative tumor markers were normal.

Two women were diagnosed with low-grade ESS; one underwent vaginal hysterectomy $(\mathrm{VH})$ for prolapse uterus, and another underwent robot-assisted myomectomy for a $6 \mathrm{~cm}$ intramural fibroid. The first patient with a prolapsed uterus had a preoperative TVS, which revealed a normal-looking endometrium with small intramural fibroids and normal bilateral ovaries, while the second patient underwent MRI, which revealed a single intramural fibroid. Following the ESS diagnosis, both women underwent re-surgery in the form of bilateral salpingo-oophorectomy in $\mathrm{VH}$ patients and TAH with BSO in the myomectomy patient, and both were found to be stage 1. The incidence of ESS in our population of hysterectomy and myomectomies was $0.3 \%$, which is in agreement with that reported in the literature $[15,16]$.

In this study, occult ovarian malignancy incidence was $0.3 \%$ in women undergoing hysterectomy with or without BSO. Similar results were reported by Desai et al. [9] in their study of 24,076 hysterectomies performed for presumed benign pathologies. There were 2 cases of ovarian malignancy in our study population. The first patient was an operated case of breast cancer breast presented to us with a chief complaint of intermenstrual bleeding. She had been taking tamoxifen for the last 2 years and was not evaluated for BRCA mutation. Her preoperative endometrial biopsy revealed proliferative endometrium, and ultrasound showed a $3 \mathrm{~cm}$ hemorrhagic cyst in the left ovary with normal CA-125; hence, advanced imaging was not obtained. Intraoperative findings were also not suggestive of any ovarian malignancy, but the postoperative examination revealed a neoplastic lesion in the left ovary likely to be metastatic. Although rare, ovaries constitute a metastatic site for primary malignancies of the breast, colon, and stomach [17]. The second case of occult ovarian malignancy was reported in a postmenopausal patient who underwent VH with BSO. Her preoperative USG was suggestive of bilateral polycystic ovaries that appeared bulky intraoperatively. A postoperative histopathology report revealed a granulosa cell tumor of the right ovary and simple hyperplasia of the endometrium. The patient was staged as $\mathrm{IA}$ and hence did not receive any postoperative chemotherapy and was disease-free at 15 months follow-up.

Cervical cancer is the only genital cancer most amenable to screening, which is almost always done during the preoperative workup prior to elective hysterectomies. The incidence of accidentally diagnosed cervical malignancy was found to be $0.15 \%$, and only one case of cervical malignancy was diagnosed postoperatively in the form of minimal deviation adenocarcinoma (MDA), also known as "Adenoma malignum." The patient was a postmenopausal woman with symptomatic fibroid uterus and underwent TLH. Preoperatively, cervical smear was normal, while intraoperatively, a long cord-like cervix was noted. Histopathology revealed MDA with more than $7 \mathrm{~mm}$ depth of invasion. Postoperative CECT did not show any pelvic or abdominal metastasis, and the patient received adjuvant radiation because MDA is considered an aggressive tumor. The patient was disease-free at the end of 20 months of follow-up using clinical examination, 6-month CECT, and vault smears.

In the largest series of occult genital tract malignancies reported by Desai et al. [9], the incidences of occult cervical, uterine and ovarian malignancy were $0.6 \%, 1.44 \%$, and $0.19 \%$, respectively, while no occult malignancies were reported in their myomectomy population. In our study, occult uterine and cervical malignancy incidence were lower $(0.47 \%$ and $0.15 \%$, respectively), while the incidence of occult ovarian malignancy was higher $(0.3 \%)$. The incidence of accidentally diagnosed malignancies was $2.8 \%$ in women undergoing myomectomy. In our study, the majority (67\%) was postmenopausal, but only $10 \%$ of them had associated comorbidities like raised $\mathrm{BMI}$, diabetes, hypertension, and prior history of pelvic pathology; however, these factors were found to have a significant association with the incidence of occult genital malignancies in the study reported by Desai et al. [9].

This study provides important insights into the incidence 


\section{Obstetrics \& Gynecology Science}

Vol. 64, No. 2, 2021

of accidentally diagnosed genital malignancies in women undergoing hysterectomy or myomectomy for presumed benign pathologies with detailed information on their preoperative workups. Although the incidence of uterine malignancies is lower than that reported in the past, it is important to suspect vicious pathologies in specific subsets of patients, such as postmenopausal women, large fibroids $(>10 \mathrm{~cm})$, and benign-like ovarian cysts in high-risk women. In the present era of minimal access surgery, more stringent preoperative screening of genital malignancies must become a norm in routine gynecologic practice. We missed one case of ESS, endometrial carcinoma, and granulosa cell tumor in some postmenopausal women, and preoperative MRI was not performed in any of them. Most gynecological pathologies are first evaluated on ultrasound, while advanced imaging techniques such as CECT or MRI are used to further investigate suspicious pathologies, although the findings of this study indicate that routine MRI must be performed in women undergoing conservative surgeries such as myomectomies, cystectomies for $>10 \mathrm{~cm}$ ovarian cysts, and in postmenopausal females with any fibroid or bulky enlarged ovaries.

\section{Conflict of interest}

No potential conflict of interest relevant to this article was reported.

\section{Ethical approval}

The Institutional Ethics Committee of All India Institute of Medical Sciences approved the study.

\section{Patient consent}

Informed consent was obtained from all patients to analyze their preoperative, intraoperative, and postoperative findings.

\section{Funding information}

None

\section{References}

1. Cohen SL, Vitonis AF, Einarsson JI. Updated hysterectomy surveillance and factors associated with minimally invasive hysterectomy. JSLS 2014;18:e2014.00096.

2. Mahnert N, Morgan D, Campbell D, Johnston C, AsSanie $S$. Unexpected gynecologic malignancy diagnosed after hysterectomy performed for benign indications. Obstet Gynecol 2015;125:397-405.

3. Rivard C, Salhadar A, Kenton K. New challenges in detecting, grading, and staging endometrial cancer after uterine morcellation. J Minim Invasive Gynecol 2012;19:313-6.

4. U.S. Food and Drug Administration. FDA safety communication. UPDATED: laparoscopic uterine power morcellation in hysterectomy and myomectomy. Silver Spring (MD): U.S. Food and Drug Administration; 2015 [cited 2020 Jun 30]. Available from: www.bogg.com/custom/ images/pdfs/MorcellationNoticeFDA04-17-2014.pdf.

5. Ramm O, Gleason JL, Segal S, Antosh DD, Kenton KS. Utility of preoperative endometrial assessment in asymptomatic women undergoing hysterectomy for pelvic floor dysfunction. Int Urogynecol J Pelvic Floor Dysfunct 2012;23:913-7.

6. Pritts EA, Vanness DJ, Berek JS, Parker W, Feinberg R, Feinberg $J$, et al. The prevalence of occult leiomyosarcoma at surgery for presumed uterine fibroids: a metaanalysis. Gynecol Surg 2015;12:165-77.

7. Hill AJ, Carroll AW, Matthews CA. Unanticipated uterine pathologic finding after morcellation during robotic-assisted supracervical hysterectomy and cervicosacropexy for uterine prolapse. Female Pelvic Med Reconstr Surg 2014;20:113-5.

8. Liu FW, Galvan-Turner VB, Pfaendler KS, Longoria TC, Bristow RE. A critical assessment of morcellation and its impact on gynecologic surgery and the limitations of the existing literature. Am J Obstet Gynecol 2015;212:71724.

9. Desai VB, Wright JD, Schwartz PE, Jorgensen EM, Fan L, Litkouhi $B$, et al. Occult gynecologic cancer in women undergoing hysterectomy or myomectomy for benign indications. Obstet Gynecol 2018;131:642-51.

10. White MP, Rahimi S, Garely A, Buhl A, Dean RM. Uterine smooth muscle tumors of uncertain malignant potential (STUMP): review of pathophysiology, classification, diag- 


\section{Obstetrics \& Gynecology Science}

Garima Yadav, et al. Incidental genital tract malignancies

nosis, treatment, and surveillance. J Healthc Commun 2017;2:40.

11. Şahin H, Karatas F, Coban G, Özen Ö, Erdem Ö, Onan $M A$, et al. Uterine smooth muscle tumor of uncertain malignant potential: fertility and clinical outcomes. J Gynecol Oncol 2019;30:e54.

12. Gadducci A, Zannoni GF. Uterine smooth muscle tumors of unknown malignant potential: a challenging question. Gynecol Oncol 2019;154:631-7.

13. Mowers EL, Skinner B, McLean K, Reynolds RK. Effects of morcellation of uterine smooth muscle tumor of uncertain malignant potential and endometrial stromal sarcoma: case series and recommendations for clinical practice. J Minim Invasive Gynecol 2015;22:601-6.

14. Ganesan R. Uterine smooth muscle tumour of uncertain malignant potential (STUMP): where are we now? Case
Rep Womens Health 2020;26:e00176.

15. Frick AC, Walters MD, Larkin KS, Barber MD. Risk of unanticipated abnormal gynecologic pathology at the time of hysterectomy for uterovaginal prolapse. Am J Obstet Gynecol 2010;202:507.e1-4.

16. Ackenbom MF, Giugale LE, Wang Y, Shepherd JP. Incidence of occult uterine pathology in women undergoing hysterectomy with pelvic organ prolapse repair. Female Pelvic Med Reconstr Surg 2016;22:332-5.

17. Rosendahl M, Timmermans Wielenga $V$, Nedergaard L, Kristensen SG, Ernst E, Rasmussen PE, et al. Cryopreservation of ovarian tissue for fertility preservation: no evidence of malignant cell contamination in ovarian tissue from patients with breast cancer. Fertil Steril 2011;95:2158-61. 